Retrospektive und Perspektive

\title{
50 Jahre Stabilitäts- und Wachstumsgesetz
}

\begin{abstract}
Als Reaktion auf die erste nennenswerte Krise im Wirtschaftswunderland Deutschland wurde am 8. Juni 1967 das Stabilitätsund Wachstumsgesetz (StWG) verabschiedet. Damit war der Siegeszug des Keynesianismus auch in Deutschland angekommen. 50 Jahre nach der Einführung ist es Zeit für eine Bilanz. Von Rudi Kurz und Joachim H. Spangenberg
\end{abstract}

P raktische stabilitätspolitische Bedeutung hatte das Stabilitäts- und Wachstumsgesetz nur für sehr kurze Zeit, weil es schon Ende der 1970er Jahre zu einem Paradigmenwechsel hin zu Monetarismus und angebotsorientierter Wirtschaftspolitik (heute oft vereinfachend „Neoliberalismus“) kam und dem neuen Weltbild folgend auch neue wirtschaftspolitische Schwerpunkte gesetzt wurden.

Obwohl viele Detailregelungen heute kaum noch bekannt sind, werden die in 『1 formulierten Ziele der Wirtschaftspolitik noch heute als Klassiker gerne zitiert: „Bund und Länder haben bei ihren wirtschafts- und finanzpolitischen Maßnahmen die Erfordernisse des gesamtwirtschaftlichen Gleichgewichts zu beachten. Die Maßnahmen sind so zu treffen, dass sie im Rahmen der marktwirtschaftlichen Ordnung gleichzeitig zur Stabilität des Preisniveaus, zu einem hohen Beschäftigungsstand und außenwirtschaftlichem Gleichgewicht bei stetigem und angemessenem Wirtschaftswachstum beitragen."

Im Gesetz werden weder konkrete Zielwerte noch Indikatoren angegeben und schon gar nicht spezifiziert, was unter "marktwirtschaftlicher Ordnung" $\mathrm{zu}$ verstehen ist, die ja als limitierender Rahmen für das staatliche Handeln genannt wird. In den 50 Jahren seit der Verabschiedung haben sich die Vorstellungen dazu und die Gewichte der einzelnen Ziele deutlich verändert.

Stabilität des Preisniveaus ist nach wie vor ein wichtiges Politikziel, dessen Durchsetzung aber erst an die Bun- desbank und dann an die EZB delegiert wurde. Während das StWG ursprünglich primär auf die Bekämpfung von Inflation zielte, interpretiert die EZB eine Inflationsrate von $2 \%$ als Preisniveaustabilität und sieht bei niedrigeren Werten Deflationsgefahren.

Hoher Beschäftigungsstand war bis Mitte der 1960er Jahre eine Arbeitslosenquote in der Größenordnung von 1\%. Auch mit dem StWG gelang es in den 1970er Jahren nicht mehr, dieses Ziel zu erreichen. Nach den Ölpreis-Schocks entstand „Stagflation“, ein Phänomen, mit dem die etablierte keynesianische Konjunkturpolitik überfordert war. Mit dem Paradigmenwechsel in den 1980er Jahren gab der Staat die Makro-Steuerung des Arbeitsmarktes auf und es kamen Strukturreformen (Deregulierung) auf die Tagesordnung.

Außenwirtschaftliches Gleichgewicht -interpretiert als ausgeglichene Handelsbilanz - hat Deutschland nie erreicht und Bundesregierungen aller Couleur waren stolz auf den Titel „Exportweltmeister". Allerdings braucht Deutschland einen Handelsbilanz-Überschuss, um die Defizite z. B. in der Dienstleistungsbilanz (Tourismus) auszugleichen und insgesamt eine ausgeglichene Leistungsbilanz zu erreichen. In den letzten Jahren haben die Überschüsse in der Handelsund in der Leistungsbilanz allerdings Dimensionen angenommen, die in der EU und bei anderen Handelspartnern Be-

\section{Veränderung der Ziele}

sorgnis hervorrufen. Der Leistungsbilanz-Überschuss liegt nun bei 7,5\% des $\mathrm{BIP}$, deutlich über den noch EU-konformen $6 \%$. Was könnte eine Bundesregierung tun? Durch den Euro ist uns der Wechselkursmechanismus abhandengekommen und die Sache erledigt sich nicht über den Devisenmarkt, d.h. durch eine Aufwertung der heimischen Währung. Daher können wir jetzt nur unsere Kosten erhöhen (um den Export zu drosseln) und/oder unsere Importe erhöhen. Kostensteigerungen könnten sich z. B. durch einen (höheren) Mindestlohn, verbesserte Sozialleistungen, höhere Infrastruktur-Investitionen oder verstärkten Klimaschutz ergeben. Allerdings ist die Modernisierung der Infrastruktur und des Energiesystems eine Investition in die Zukunft und wird die Wettbewerbsfähigkeit Deutschlands schließlich weiter steigern. Um höhere Importe zu erreichen, müsste geprüft werden, ob nicht tarifäre Handelshemmnisse abgebaut werden können (Genfood etc.) oder wie die Binnennachfrage weiter gesteigert werden könnte (Mehrkonsum) - beides ist unter Nachhaltigkeitsaspekten wenig sinnvoll.

\section{Das magische Viereck}

Stetiges und angemessenes Wirtschaftswachstum hat sich unter den vier Zielen eine Führungsrolle erobert. Faktisch ist damit heute das "magische Viereck" aufgehoben und Wachstum ist das Oberziel, das zugleich einen hohen Beschäftigungsstand sichern soll. Weiter wird davon ausgegangen, dass es keinen Konflikt mit der Preisniveaustabilität gibt (bei geeigneter Notenbankpolitik) und dass Wachstum das außenwirtschaftliche Gleichgewicht nicht stört (bei flexiblen Wechselkursen). Auf der Grundlage dieser Prämissen ist Wirtschaftspolitik heute im Wesentlichen Wachstumspolitik: Alle Politikbereiche stehen unter dem Wachstumsprimat und werden daran ausgerichtet bzw. unter Wachstumsvorbehalt gestellt - von der Energieüber die Verkehrs- bis zur Forschungspolitik. Durch die Dominanz des Wachstumsziels wäre es besonders wichtig zu 
klären, was „angemessen“ bedeutet. Aber dazu gibt das altehrwürdige StWG nichts her. „Angemessen“ heißt jedenfalls nicht „so viel wie möglich“, sondern bezieht sich vor allem auf die Produktivitätszuwächse. Wenn diese bei 1-2\% p.a. liegen, muss auch das reale BIP-Wachstum in dieser Größenordnung liegen, damit das Beschäftigungsvolumen erhalten bleibt.

\section{Neue Ziele}

In den letzten 50 Jahren sind neue Ziele für die Wirtschaftspolitik hinzugekommen, die in einem erweiterten „magischen Vieleck“ zu berücksichtigen wären:

I Schutz der natürlichen Lebensgrundlagen (Art. 20a GG, eingefügt 1994): Wirtschaftswachstum ist dann nicht angemessen, wenn dadurch Umweltbelastung und Ressourcenverbrauch weiter ansteigen und es zu einer Belastung zukünftiger Generationen kommt.

- Haushaltsausgleich (Schuldenbremse, Art. 146 GG u. a., eingefügt 2009): Ein Erbe, das sich durch keynesianisches Deficit Spending, durch Wiedervereinigung und durch Finanzkrise aufgebaut hat, ist ein Schuldenberg von 2.000 Mrd. €. Daher ist der Staatsverschuldung ein Verfassungsriegel vorgeschoben worden - selbst wenn sie der Investition und damit höherem Wachstum dienen sollte. Ausnahmen bei konjunkturellen Abweichungen von der Normallage sind bis zu 1,5\% des Bruttoinlandsprodukts zulässig (Art. 115 GG).

- Gerechtigkeit: Zwar ist in Art. 20 GG festgehalten, dass Deutschland ein sozialer Bundesstaat ist und haben die ordo-liberalen Gründungsväter die Bedeutung des sozialen Ausgleichs stets betont, doch war dies schon im StWG unberücksichtigt geblieben (anders als im Sachverständigenrats-Gesetz von 1963). Wenn aber (mehr) Wachstum nicht dazu beiträgt, vor allem die Lage der unteren Einkommensschichten $\mathrm{zu}$ verbessern, sondern primär die Reichen reicher macht, ist es nicht „angemessen“ - und verursacht ein Legitimationsproblem für die marktwirtschaftliche Ordnung insgesamt.

\section{Perspektiven}

Was also tun mit diesem Gesetz? Es gibt zwei Optionen:

- Man lässt es weiter friedlich ruhen (und betreibt im Kern Wachstumspolitik).

- Man nimmt es zum Anlass für eine Reformdebatte.

Für Option 2 würde sicherlich Einiges sprechen, weil das Zielsystem der Wirtschaftspolitik wenig transparent ist und seine Ausformulierung mit den Veränderungen der letzten Jahrzehnte nicht Schritt gehalten hat. Ansatzweise kann die Deutsche Nachhaltigkeitsstrategie (Neuauflage 2016) als ein Versuch in diese Richtung verstanden werden, der auch die globale Entwicklung berücksichtigt (UN Sustainable Development Goals).

Die Festlegung von Zielen und Indikatoren kann nur ein erster Schritt zu einer Strategie sein, die auch Zielkonflikte analysiert, Maßnahmen und Ressourcen definiert. Zudem ist die Nachhaltigkeitsstrategie lediglich ein Kabinettsbeschluss und hat - anders als das StWG noch immer keine Gesetzeskraft. Das sind schon skurrile Verhältnisse, dass eine weitgehend obsolete Vorstellung von Konjunktur- und Wachstumspolitik gesetzlich geregelt ist, während wir die Zukunftsfähigkeit als Verwaltungsakt behandeln.

\section{AUTOREN + KONTAKT}

Dr. Rudi Kurz ist Professor für Volkswirtschaftslehre an der Fakultät für Wirtschaft und Recht der Hochschule Pforzheim.

Hochschule Pforzheim, Tiefenbronner Str. 65, 75175 Pforzheim. Tel.: +497231 286105.

Joachim H.Spangenberg ist Wissenschaftler am Sustainable Europe Research Institute SERI Germany e. V.

Sustainable Europoe Research Institute SERI Germany e. V., Vorsterstr. 97-99, 51103 Köln.

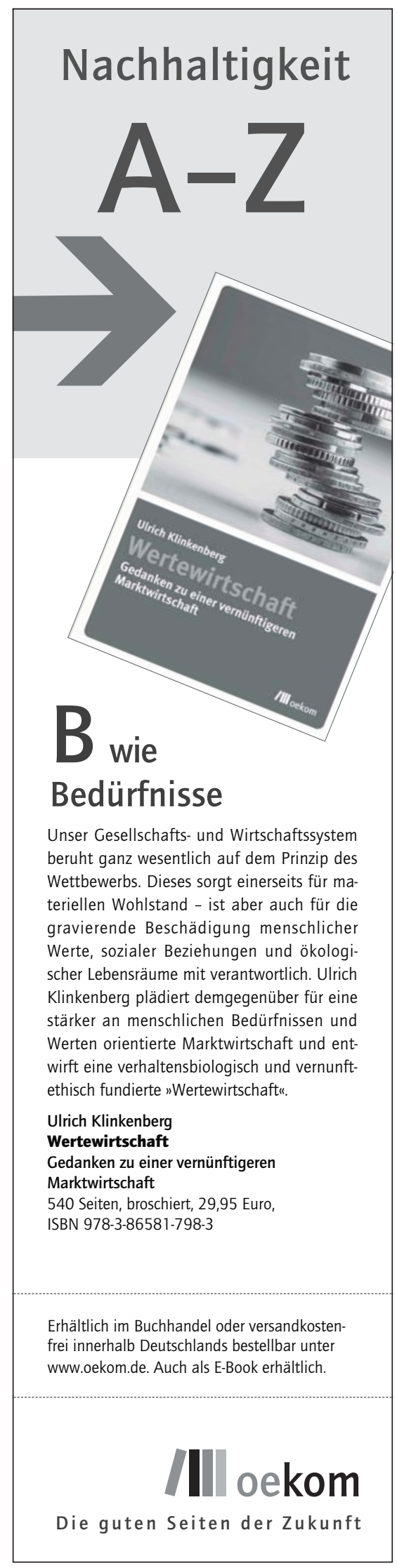

